\title{
Quantum Wave Functionals and Some Related Topics
}

\author{
J. G. GILSON \\ Mathematics Department, Queen Mary College, London \\ (Z. Naturforsch. 23a, 1452-1458 [1968]; received 23 May 1968)
}

\begin{abstract}
This paper is concerned, in the first place, with showing how a formula derived by the present author in an earlier paper can be given a more satisfactory form and definition. Secondly, some of the properties of this formula and difficulties connected with its possible use are discussed. In connection with this work the concept of "Quantum Probability Pressure" is used and it is shown that this makes an analogy with fluid dynamics complete.
\end{abstract}

We shall be concerned with showing how a formula (reference ${ }^{1}$, Eqs. (III, 17, 29)) can be given a more satisfactory form, definition and interpretation. The formula to which we refer is,

$$
\Psi\left(x_{\mathrm{B}}, t_{\mathrm{B}}\right)=\exp \left\{\frac{i}{\hbar} \int_{t_{\mathrm{A}}}^{t_{\mathrm{B}}}\left[\frac{P^{2}(x(t), t)}{2 m}+\frac{i \hbar}{2 m} \frac{\partial P(x(t), t)}{\partial x(t)}-W(x(t), t)\right] \mathrm{d} t\right\} \Psi\left(x_{\mathrm{A}}, t_{\mathrm{A}}\right) .
$$

This formula connects the wave function, $\Psi\left(x, t_{\mathrm{A}}\right)$, at time $t_{\mathrm{A}}$ with the wave function, $\Psi\left(x, t_{\mathrm{B}}\right)$, at time $t_{\mathrm{B}}$ under the influence of a prescribed external potential $W(x, t)$. The integration in (1) is along a path, $x(t)$, such that

$$
\begin{gathered}
\frac{\mathrm{d} x(t)}{\mathrm{d} t}=\frac{P(x(t), t)}{m}, \\
x\left(t_{\mathrm{A}}\right)=x_{\mathrm{A}} \text { and } x\left(t_{\mathrm{B}}\right)=x_{\mathrm{B}} .
\end{gathered}
$$

At first sight formula (1) looks a very promising computational tool for finding the wave function at time $t_{\mathrm{B}}$ if it is known at time $t_{\mathrm{A}}$. On closer examination one finds that it is, in fact, of very little use in this respect; for in order to make use of it one would have to know quite a lot about $P(x, t)$ between times $t_{\mathrm{A}}$ and $t_{\mathrm{B}}$. Thus since

$$
P(x, t)=-i \hbar \frac{\partial \log \psi(x, t)}{\partial x},
$$

this would be tantamount to already knowing much about $\Psi(x, t)$ for $t>t_{\mathrm{A}}$, which is, after all, what one is trying to find. However, formula (1) and a similar formula which we shall derive later does tell us something about the under-lying structure of Quantum mechanics. For one thing it showsclearly how the functional integral from which it was derived can always be evaluated for any potential $W(x, t)$. The exponential of a functional which appears in Eq. (1) is, indeed, the result of performing

1 J. G. Gilson, Nuovo Cim. (Ser. X) 40, 993 [1965].

2 N. Wiener, J. Math. Phys. 2. 131 [1923].

3 R. P. Feynman, Rev. Mod. Phys. 29, 367 [1948]. this integration. We shall use the term "functional integral" as meaning an integration over a function space such as is a Wiener integral ${ }^{2}$ or as is a Feynman integral ${ }^{3}$. The functional integral should not be confused with the integral of a functional which appears in the exponent of formula (1) and which, once the path is settled, only depends on the one function for this path. Integrals such as that which appears in the exponent of (1), we shall call contour integrals. The functional in the exponent of $(1)$ is clearly closely related to a classical contact transformation (see references ${ }^{4}$ and ${ }^{5}$ ) and so we see how upon integration the functional integral converts into an exponential of a contact transformation. A more subtle hope for using (1) computationally would be to employ some special path. It is tempting to try classical dynamical paths for the potential $W(x, t)$, assuming such paths can be calculated. We soon find that for one thing we are in trouble with the derivative term $\partial P / \partial x$, which we have no means of calculating. As it happens the paths of importance in this work are not classical dynamical paths at all.

They actually satisfy the differential equation (ref. 1, IV 4)

$$
\frac{\mathrm{d} P}{\mathrm{~d} t}=-\frac{\partial}{\partial x}\left[-\frac{i \hbar}{2 m} \frac{\partial P}{\partial x}+W\right]
$$

where, incidentally, $P$ is a complex function. Thus if we try to calculate the solutions to (4), we are

4 E. T. Whittaker, A treatise on the analytical dynamics of particles and rigid bodies, Cambridge University Press, Fourth Edition 1960, p. 38 and 245.

5 P. A. M. Dirac, The principles of Quantum Mechanics, Oxford University Press, Third Edition 1947, p. 128. 
still in trouble with the $(-i \hbar / 2 m) \partial P / \partial x$ term which is an addition to the classical potential $W(x, t)$ and can be seen to be a sort of feed back effect in which neighbouring paths cause a disturbance to the path of immediate interest.

In this paper we do not claim to be able to invent a technique which makes computation with (1) possible, but we are able to go some way in clarifying the basic significance of (1). We shall also show that with a small reinterpretation of earlier work, the analogy with fluid motion (see reference ${ }^{1}$ p. 14) can be made completely realistic by the introduction of a "probability pressure".

The problems of interpreting and manipulating functional integrals are many, difficult and interrelated. The result is that the complexity and confusion often seems to make any attempt to work within this area of ideas almost impossible and very discouraging. However, one can concentrate on solving parts of this general area of problem and hope that with the passage of time, the whole will come within the scope of some mathematical technique or other. It is with this attitude in mind we here deal with the one aspect of defining our basic functional and its two derivatives and the other aspect of extending the fluid motion analogy.

\section{The Transition Wave Functional}

Many of the problems connected with functional integration and the use of functionals in general are as acute in one dimension as they are in higher numbers of dimensions so that here, as elsewhere ${ }^{1,6}$ when we have studied these problems, the one dimensional case only will be considered. Thus we shall study a system which is confined to a line and which has characteristics changing with time.

Consider then a vector function, $\boldsymbol{F}(x, t)$, which is a gradient in the $(x, t)$ plane.

$$
\boldsymbol{F}(x, t)=\boldsymbol{k} \frac{\partial \chi}{\partial x}+\boldsymbol{j} \frac{\partial \chi}{\partial t} .
$$

The vectors $\boldsymbol{j}$ and $\boldsymbol{k}$ are of unit length and point along the time and space directions respectively. The question of a metric in this space time plane is not important. We shall simply regard this plane as being a map for describing certain functions and paths and so it can be taken to be Euclidean. We

6 J. G. Gilson, Quantum Probability Weighted Paths, To be published. shall denote the components of $\boldsymbol{F}$ by $+P(x, t)$ and $-E(x, t)$. The sign preceding the function $E$ is just a matter of convenience for later interpretation.

Thus

$$
\boldsymbol{F}=P \boldsymbol{k}-E \boldsymbol{j}
$$

and

$$
\operatorname{Curl} \boldsymbol{F}=\nabla \times \nabla \boldsymbol{\chi} \equiv \mathbf{0} .
$$

It follows from (7) by Stokes' theorem that the line integral of $\boldsymbol{F}$ taken round a closed contour $C$ is identically zero, provided that $C$ lies in a region $\mathrm{R}$ where $\boldsymbol{F}$ has the necessary conditions of finiteness and continuity ${ }^{7}$. Hence when we work in such a region, $\mathrm{R}$, we have

where

$$
\oint_{C} \boldsymbol{F}(x(t), t) \mathrm{d} \boldsymbol{u}(t)=0
$$

$\boldsymbol{u}(t)=x(t) \boldsymbol{k}+t \boldsymbol{j} \quad$ or $\quad \mathrm{d} \boldsymbol{u}(t)=\mathrm{d} x(x) \boldsymbol{k}+\mathrm{d} t \boldsymbol{j}$

and $x(t)$ is a path lying entirely within $\mathrm{R}$ and is denoted by the letter $C$. We shall restrict the discussion here to paths, $x(t)$, which are differentiable with respect to time because in the quantum domain where we wish to make applications of this work such paths are all important. This contrasts sharply with the Brownian motion case where the path derivatives are mostly infinite (see ref. ${ }^{2}$ and ${ }^{6}$ ).

We now consider two distinct points (events) on the contour $C$. Let these be $\left(x\left(t_{\mathrm{A}}\right), t_{\mathrm{A}}\right)$ and $\left(x\left(t_{\mathrm{B}}\right), t_{\mathrm{B}}\right)$ and suppose that they divide the contour $C$ into two parts $C_{1}$ and $C_{2}$. Thus we get from (8) the relation,

$$
\int_{C_{1}} \boldsymbol{F}\left(x_{1}(t), t\right) \mathrm{d} \boldsymbol{u}_{1}(t)=\int_{-C_{2}} \boldsymbol{F}\left(x_{2}(t), t\right) \mathrm{d} \boldsymbol{u}_{2}(t)
$$

where now $x_{1}(t)$ and $x_{2}(t)$ are the functions representing the separate parts $C_{1}$ and $-C_{2}$ respectively. The minus sign represents a path described in the reverse direction. From (10), we see that for all contours, within $\mathrm{R}$, joining $\left(x\left(t_{\mathrm{A}}\right), t_{\mathrm{A}}\right)$ to $\left(x\left(t_{\mathrm{B}}\right), t_{\mathrm{B}}\right)$ our integral in (10) will have the same value. We now wish to define a form of differentiation of integrals such as those which appear in (10). These integrals, as we have seen, depend on path end points and not on the path, provided the path does not go outside a suitable region $R$. We shall therefore use the notation

$$
\Phi\left(x\left(t_{\mathrm{B}}\right), t_{\mathrm{B}} \mid x\left(t_{\mathrm{A}}\right), t_{\mathrm{A}}\right)=\int_{C} \boldsymbol{F}(x(t), t) \mathrm{d} \boldsymbol{u}(t),
$$

7 L. M. Mulne-Thomson, Theoretical Hydrodynamics, MacMillan, Second Edition 1949, p. 81. 
with $C$ being any suitable path connecting $\mathrm{A}$ and $\mathrm{B}$. We now intend to define derivatives of $\Phi$ with respect to $t_{\mathrm{B}}$ and $x\left(t_{\mathrm{B}}\right)$ regarded as independent variables. If we use (5), (6) and (9), we see that (11) can be expressed in the form,

$\Phi\left(x\left(t_{\mathrm{B}}\right), t_{\mathrm{B}} \mid \mathrm{A}\right)=\int_{x\left(t_{\mathrm{A}}\right)}^{x\left(t_{\mathrm{B}}\right)} P(x(t), t) \mathrm{d} x(t)-\int_{t_{\mathrm{A}}}^{t_{\mathrm{B}}} E(x(t), t) \mathrm{d} t$,

where also we have used the condensed notation, $\left.\mid \mathrm{A}) \equiv \mid x\left(t_{\mathrm{A}}\right), t_{\mathrm{A}}\right)$. From (12) we see that a superficial answer to what the two derivatives should be is,

$$
\frac{\partial \Phi\left(x\left(t_{\mathrm{B}}\right), t_{\mathrm{B}} \mid \mathrm{A}\right)}{\partial t_{\mathrm{B}}}=-E\left(x\left(t_{\mathrm{B}}\right), t_{\mathrm{B}}\right),
$$

and $\frac{\partial \Phi\left(x\left(t_{\mathrm{B}}\right), t_{\mathrm{B}} \mid \mathrm{A}\right)}{\partial x\left(t_{\mathrm{B}}\right)}=P\left(x\left(t_{\mathrm{B}}\right), t_{\mathrm{B}}\right), \quad(\mathrm{b})$.

Equations $(13 \mathrm{a}, \mathrm{b})$ are, in fact, the correct answer. However, a little thought will raise, among other questions, the uncertainty of what effect a change in $x\left(t_{\mathrm{B}}\right)$ at the fixed time $t_{\mathrm{B}}$ will have particularly if one wishes to keep the varied path continuous up to $t_{\mathrm{B}}$.

In that case $x(t)$ will have to change for all $t$ in some small region before up to and including $t_{\mathrm{B}}$. In which case this spread variation in $x(t)$ will lead to a more complicated change in (12) in which $E(x(t), t)$ for $t \leqq t_{\mathrm{B}}$ varies. Thus one might expect that varying $x\left(t_{\mathrm{B}}\right)$ will lead to contributions from the $E$ term.

The reader who doubts the non triviality of these difficulties might express (12) as a Riemann sum and then see what happens when $x(t)$ is varied in a small region before $t_{\mathrm{B}}$. It is the case that the resolution of these uncertainties is inherent in the way we have expressed the problem and this we shall now show in detail.

\section{The Time Derivative}

To define the time derivative we shall use a contour of the shape shown in diagram 1.

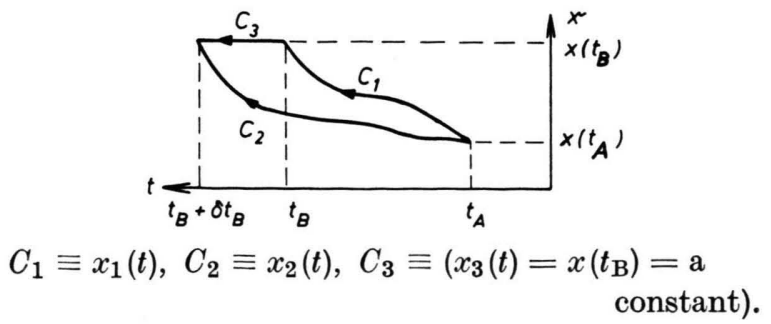

The important feature of this contour $C$, which is composed of sections $C_{1}, C_{2}$ and $C_{3}$ and is such that $C_{1}+C_{3}=C_{2}$, is the horizontal section $C_{3}$ between $t_{\mathrm{B}}$ and $t_{\mathrm{B}}+\delta t_{\mathrm{B}}$. Thus the contour $C_{1}$ can be regarded as having been shifted and possibly deformed into the position $C_{2}$ by displacement parallel to the time axis. The shifting contour is kept continuous and the point $\left(x\left(t_{\mathrm{A}}\right), t_{\mathrm{A}}\right)$ remains fixed.

To define our time derivative we shall use the form (11) and employ its variation under the transformation $C_{1} \rightarrow C_{2}$ as illustrated in diagram 1 . Thus we have

$$
\begin{array}{r}
\frac{\partial \Phi(\mathrm{B} \mid \mathrm{A})}{\partial t_{\mathrm{B}}}=\lim _{\delta t_{\mathrm{B}} \rightarrow 0} \frac{1}{\delta t_{\mathrm{B}}}\left[\int_{C_{2}} \boldsymbol{F}\left(x_{2}(t), t\right) \mathrm{d} \boldsymbol{u}_{2}(t)\right. \\
\left.-\int_{C_{1}} \boldsymbol{F}\left(x_{1}(t), t\right) \mathrm{d} \boldsymbol{u}_{1}(t)\right] \\
=\lim _{\delta t_{\mathrm{B}} \rightarrow 0} \frac{1}{\delta t_{\mathrm{B}}} \int_{C_{3}} \boldsymbol{F}\left(x_{3}(t), t\right) \mathrm{d} \boldsymbol{u}_{3}(t) .
\end{array}
$$

This is because $C_{2}-C_{1}-C_{3}=0$ gives a closed contour for which (8) is true. Now on the straight section $C_{3}, \mathrm{~d} \boldsymbol{u}_{3}(t)=\boldsymbol{j} \mathrm{d} t$ and $x_{3}(t)=x\left(t_{\mathrm{B}}\right)$. Therefore

$$
\begin{aligned}
\frac{\partial \Phi(\mathrm{B} \mid \mathrm{A})}{\partial t_{\mathrm{B}}} & =-\lim _{\delta t_{\mathrm{B}} \rightarrow 0} \frac{1}{\delta t_{\mathrm{B}}} \int_{t_{\mathrm{B}}}^{t_{\mathrm{B}}+\delta t_{\mathrm{B}}} E\left(x\left(t_{\mathrm{B}}\right), t\right) \mathrm{d} t \\
& =-E\left(x\left(t_{\mathrm{B}}\right), t_{\mathrm{B}}\right) .
\end{aligned}
$$

Thus we do get the time differentiation formula (13a) unambiguously.

\section{The Space Derivative}

To justify the formula ( $13 \mathrm{~b}$ ) we use the contour shown in diagram 2.

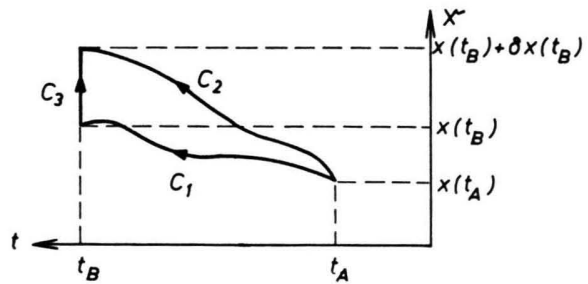

$$
C_{1} \equiv x_{1}(t), C_{2} \equiv x_{2}(t), C_{3} \equiv\left(t_{\mathrm{B}}=\text { a constant }\right) .
$$

The important feature of this contour $C$, which is composed of sections $C_{1}, C_{2}$ and $C_{3}$ and is such that $C_{1}+C_{3}=C_{2}$, is the vertical section between $x\left(t_{\mathrm{B}}\right)$ and $x\left(t_{\mathrm{B}}\right)+\delta x\left(t_{\mathrm{B}}\right)$. Thus in this case the contour $C_{1}$ can be regarded as having been shifted and possibly deformed into the position $C_{2}$ by displacement par- 
allel to the $x$ axis. The shifting contour is kept continuous and the point $\left(x\left(t_{\mathrm{A}}\right), t_{\mathrm{A}}\right)$ remains fixed.

We now define the space derivative of $\Phi$ by

$$
\begin{array}{r}
\frac{\partial \Phi(\mathrm{B} \mid \mathrm{A})}{\partial x\left(t_{\mathrm{B}}\right)}=\lim _{\delta x\left(t_{\mathrm{B}}\right) \rightarrow 0} \frac{1}{\delta x\left(t_{\mathrm{B}}\right)}\left[\int_{C_{2}} \boldsymbol{F}\left(x_{2}(t), t\right) \mathrm{d} \boldsymbol{u}_{2}(t)\right. \\
\left.-\int_{C_{1}} \boldsymbol{F}\left(x_{1}(t), t\right) \mathrm{d} \boldsymbol{u}_{1}(t)\right] \\
=\lim _{\delta x\left(t_{\mathrm{B}}\right) \rightarrow 0} \frac{1}{\delta x\left(t_{\mathrm{B}}\right)} \int_{C_{3}} \boldsymbol{F}\left(x_{3}(t), t\right) \mathrm{d} \boldsymbol{u}_{3}(t) .
\end{array}
$$

On the section $C_{3}, t=t_{\mathrm{B}}=$ constant, and $\mathrm{d} \boldsymbol{u}_{3}(t)$ $=\boldsymbol{k} \mathrm{d} x\left(t_{\mathrm{B}}\right)$. Therefore

$$
\begin{aligned}
\frac{\partial \Phi(\mathrm{B} \mid \mathrm{A})}{\partial x\left(t_{\mathrm{B}}\right)} & =\lim _{\delta x\left(t_{\mathrm{B}}\right) \rightarrow 0} \frac{1}{\delta x\left(t_{\mathrm{B}}\right)} \int_{x\left(t_{\mathrm{B}}\right)}^{\left.x\left(t_{\mathrm{B}}\right)+\delta x\left(t_{\mathrm{B}}\right)\right]} P\left(x\left(t_{\mathrm{B}}\right), t_{\mathrm{B}}\right) \mathrm{d} x\left(t_{\mathrm{B}}\right) \\
& =P\left(x\left(t_{\mathrm{B}}\right), t_{\mathrm{B}}\right)
\end{aligned}
$$

and so we have the justification of $(9, b)$. Thus we have a unique pair of derivatives for our functional $\Phi(\mathrm{A} \mid \mathrm{B})$ at the end point A and clearly similar derivatives could be defined for the end point $B$.

The exponential of the functional $(i / \hbar) \Phi(\mathrm{B} \mid \mathrm{A})$ will be referred to as the transition wave functional and will be denoted by the symbol

$$
\Psi(\mathrm{B} \mid \mathrm{A})=\exp (i / \hbar) \Phi(\mathrm{B} \mid \mathrm{A}) .
$$

Thus using (12) we have

$\Psi(\mathrm{B} \mid \mathrm{A})=\exp \left\{\frac{i}{\hbar}\left[\int_{x\left(t_{\mathrm{A}}\right)}^{x\left(t_{\mathrm{B}}\right)} P(x(t), t) \mathrm{d} x(t)-\int_{t_{\mathrm{A}}}^{t_{\mathrm{B}}} E(x(t), t) \mathrm{d} t\right]\right\}$

\section{Amplitude of the Wave Functional}

Before going on to consider equations of motion, we shall now show how the transition wave functional (16) converts the wave function $\Psi(\mathrm{A}) \equiv$ $\equiv \Psi\left(x, t_{\mathrm{A}}\right)$ into the wave function $\Psi(\mathrm{B}) \equiv \Psi\left(x, t_{\mathrm{B}}\right)$ and hence the probability density $\varrho\left(x, t_{\mathrm{A}}\right)$ into the probability density $\varrho\left(x, t_{\mathrm{B}}\right)$. We have

$$
\begin{aligned}
\Psi\left(x_{\mathrm{B}}, t_{\mathrm{B}}\right) & =\varrho^{1 / 2}(\mathrm{~B}) \exp \{i \theta(\mathrm{B})\} \\
& =\exp \{(i / \hbar) \Phi(\mathrm{B} \mid \mathrm{A})\} \Psi\left(x_{\mathrm{A}}, t_{\mathrm{A}}\right) \\
& =\exp \{i / \hbar) \Phi(B / A)\} \varrho^{1 / 2}(\mathrm{~A}) \exp \{i \theta(\mathrm{A})\}
\end{aligned}
$$

where $\theta(\mathrm{A})$ and $\theta(\mathrm{B})$ are real functions.

Thus

$$
\begin{aligned}
\varrho^{1 / 2}(\mathrm{~B}) & =\exp \{-(\mathrm{l} / \hbar) \operatorname{Im} \Phi(\mathrm{B} \mid \mathrm{A})\} \varrho^{1 / 2}(\mathrm{~A}) \\
& =\exp \left\{-(1 / \hbar) \int_{C} \operatorname{Im} \boldsymbol{F} \mathrm{d} u\right\} \varrho^{1 / 2}(\mathrm{~A})
\end{aligned}
$$

Now, we recall that $\boldsymbol{F}$ is by definition a gradient of a function $\chi$. Thus if we take the imaginary part, $\operatorname{Im} \boldsymbol{F}$, to be the gradient of $-\frac{1}{2} \hbar \log \varrho$, which follows because

$$
P_{2}=-\frac{\hbar}{2 \varrho} \frac{\partial \varrho}{\partial x} \quad \text { and } \quad E_{2}=\frac{\hbar}{2 \varrho} \frac{\partial \varrho}{\partial t}
$$

(see ref. 6, Eqs. (43) and (53)), then

$$
\begin{aligned}
\varrho^{1 / 2}(\mathrm{~B}) & =\exp \left\{+\frac{1}{2} \int_{C} \nabla \log \varrho \mathrm{d} \boldsymbol{u}\right\} \varrho(\mathrm{A}) \\
& =\left(\varrho^{1 / 2}(\mathrm{~B}) / \varrho^{1 / 2}(\mathrm{~A})\right) \varrho^{1 / 2}(\mathrm{~A}) .
\end{aligned}
$$

Thus we see how by pure multiplication the transition wave functional converts the density raised to the half power at time $\mathrm{A}$ into the density raised to a half power at time $B$.

\section{Equations of Motion}

Having defined the transition wave functional, $\Psi(\mathrm{B} \mid \mathrm{A})$ and the rules for differentiating it with respect to space and time, we now have to specify its "equation of motion". The equation of motion, as one might expect, is a functional differential equation expressing a relation between $E(x, t)$ and $P(x, t)$. We are free to choose this relation according to the properties we wish our system to have. One such choice would be that our system is to have the same properties as a quantum mechanical system described by the Schrödinger equation. In that case $\Psi(\mathrm{B} \mid \mathrm{A})$ must contain the same information as a Schrödinger wave function. This is achieved by imposing on $\Psi(\mathrm{B} \mid \mathrm{A})$ the functional differential equation,

$$
\begin{aligned}
\frac{i \hbar \partial \Psi(\mathrm{B} \mid \mathrm{A})}{\partial t_{\mathrm{B}}}= & -\frac{\hbar^{2}}{2 m} \frac{\partial^{2} \Psi(\mathrm{B} \mid \mathrm{A})}{\partial x\left(t_{\mathrm{B}}\right)^{2}} \\
& +W\left(x\left(t_{\mathrm{B}}\right), t_{\mathrm{B}}\right) \Psi(\mathrm{B} \mid \mathrm{A})
\end{aligned}
$$

and this implies the functional equation

$$
\begin{aligned}
E\left(x\left(t_{\mathrm{B}}\right), t_{\mathrm{B}}\right)= & \frac{P^{2}\left(x\left(t_{\mathrm{B}}\right), t_{\mathrm{B}}\right)}{2 m}-\frac{i \hbar}{2 m} \frac{\partial P\left(x\left(t_{\mathrm{B}}\right), t_{\mathrm{B}}\right)}{\partial x\left(t_{\mathrm{B}}\right)} \\
& +W\left(x\left(t_{\mathrm{B}}\right), t_{\mathrm{B}}\right) .
\end{aligned}
$$

Functions $E(x, t)$ and $P(x, t)$ which satisfy the ordinary equation,

$$
E(x, t)=\frac{P^{2}(x, t)}{2 m}-\frac{i \hbar}{2 m} \frac{\partial P(x, t)}{\partial x}+W(x, t)
$$

in the two independent variables $x$ and $t$ will also satisfy (20). Eq. (21), on the other hand, is the Schrödinger equation for $\Psi(x, t)$ when

$$
E=i \hbar \frac{\partial \log \Psi}{\partial t} \quad \text { and } \quad P=-i \hbar \frac{\partial \log \Psi}{\partial x} .
$$


In this way we see the relation between our functional formalism and conventional quantum mechanics. We shall next briefly consider an alternative choice for the relation between $E$ and $P$.

\section{Classical Contact Transformation}

We shall now consider what condition should be imposed on the wave functional in order that it describes a classical dynamical system. This is achieved by imposing on $\Psi(\mathrm{B} \mid \mathrm{A})$ a non linear "Schrödinger like" equation. If we work directly with the functions $E(x, t)$ and $P(x, t)$ it will be sufficient for us to use the Schrödinger equation (21) with what might be called an additional "Feed back" potential, $W_{\mathrm{F}}$.

This will imply the existence of a system which has a density state function $\varrho(x, t)$ which is capable of causing a local influence on the paths taken by particles. $\varrho(x, t)$ will as usual have the form

$$
\varrho(x, t)=\Psi^{*}(x, t) \Psi(x, t)
$$

and we shall take $W_{\mathrm{F}}$ to have the rather odd form

$$
-W_{\mathrm{F}}=\frac{\hbar^{2}}{4 m}\left[\frac{1}{2}\left(\frac{1}{\varrho} \frac{\partial \varrho}{\partial x}\right)^{2}-\left(\frac{1}{\varrho} \frac{\partial^{2} \varrho}{\partial x^{2}}\right)\right] .
$$

The significance of this choice will be clearer later. Thus our non-linear Schrödinger equation becomes

$$
i \hbar \frac{\partial \Psi}{\partial t}=-\frac{\hbar^{2}}{2 m} \frac{\partial^{2} \Psi}{\partial x^{2}}+\left(W+W_{\mathrm{F}}\right) \Psi
$$

We easily confirm that this leads to the two equations

$$
\begin{array}{lcc} 
& E_{1}=\frac{P_{1}^{2}}{2 m}+W \\
\text { and } & \frac{\partial \varrho}{\partial t}=-\nabla \cdot\left(\frac{P_{1}}{m} \varrho\right) \\
& \text { where } & E_{1}+i E_{2}=i \hbar \frac{\partial \log \Psi}{\partial t} \\
\text { and } & P_{1}+i P_{2}=-i \hbar \frac{\partial \log \Psi}{\partial x} .
\end{array}
$$

We see that (25) and (26) represent a pure classical situation with density satisfying the equation of continuity (26) and flowing along classical trajectories given by $E_{1}=$ constant under the influence of $W(x, t)$.

Equations (25) and (26) represent a situation in which the probabilistic aspects of the problem as described by $\varrho(x, t)$ and the dynamical aspects as

8 E. Madelung, Z. Phys. 40, 332 [1926].

9 D. Вонм and J. P. Vigier, Phys. Rev. 96, 208 [1954]. described by (25) are almost completely decoupled. The function $\left(P_{1} / m\right)$ which is essentially a classical velocity can be regarded as a velocity field which drives the density distribution via Eq. (26). This decoupling is achieved by introducing into the quantum mechanical description the feed back potential (23). In this case our transformation function $\Phi(\mathrm{B} \mid \mathrm{A})$ is essentially a classical contact transformation. This all suggests strongly that the quantum process is basically classical except for a "self-effect". That is a distribution of essentially classical paths are described but with a mutual interaction. Such a situation is surely classical hydrodynamics as opposed to classical dynamics.

We have partly considered the hydrodynamical analogy in reference ${ }^{1}$. The first introduction of this analogy appears to be due to MADELUNG ${ }^{8}$. His work was then extended by Вонм and VIGIER ${ }^{9}$. In the rest of this paper, we shall extend this point of view further using the concept of "Quantum Probability Pressure" to show that it gives a completely realistic picture of the quantum process.

\section{Probability Pressure}

In the case of a compressible fluid, when the pressure, $\pi$, is a function of the density, $\varrho$, the equation of motion for one dimensional flow (see ref. ${ }^{7}$ ) is,

$$
\frac{\partial}{\partial x}\left(\frac{\partial \beta}{\partial t}\right)=\frac{1}{\varrho} \frac{\partial \pi}{\partial x}+\frac{\partial}{\partial x}\left(\frac{1}{2} v^{2}+\Omega\right) .
$$

$\pi$ is the pressure and $\beta$ is the velocity potential such that $v=-\nabla \beta$. We can integrate (29) from $-\infty$ up to $x$ to obtain

$$
\left[\frac{\partial m \beta}{\partial t}-\frac{m v^{2}}{2}-m \Omega\right]=\int_{-\infty}^{x} \frac{m}{\varrho} \frac{\partial \pi}{\partial x} \mathrm{~d} x^{\prime} .
$$

In (30) we have also multiplied through by the constant $m$. Let us denote the quantity in square brackets in (30) by $E_{\mathrm{Q}}(x, t)$ and then (30) becomes

$$
\begin{aligned}
& E_{\mathrm{Q}}(x, t)=\frac{\partial m \beta}{\partial t}-\frac{m v^{2}}{2}-m \Omega \\
& =E_{\mathrm{Q}}(-\infty, t)+\int_{-\infty}^{x} \frac{m}{\varrho} \frac{\partial \pi}{\partial x^{\prime}} \mathrm{d} x^{\prime} .
\end{aligned}
$$

We now intend to compare (31) with the real part of Eq. (21). This real part is

$$
\frac{\partial}{\partial t}(-\hbar \operatorname{Im} \log \Psi)-\frac{P_{1}^{2}}{2 m}-W=\frac{\hbar}{2 m} \frac{\partial P_{2}}{\partial x}-\frac{P_{2}^{2}}{2 m} .
$$


Thus we can identify

$$
\begin{array}{ll}
W=m \Omega, & \mathrm{a} \\
P_{\mathbf{1}}=m v, & \mathrm{~b}
\end{array}
$$

and $-\hbar \operatorname{Im} \log \Psi=m \beta$.

c

Further to make the analogy complete, we need to put

$E_{\mathbf{Q}}(-\infty, t)+\int_{-\infty}^{x} \frac{m}{\varrho} \frac{\partial \pi}{\mathrm{d} x^{\prime}} \mathrm{d} x^{\prime}=\frac{\hbar}{2 m} \frac{\partial P_{2}}{\partial x}-\frac{P_{2}^{2}}{2 m}$.

Hence, if we assume that $E_{\mathbf{Q}}(-\infty, t)$ does not depend on $x$, we get for the pressure gradient due to the potential $W_{\pi}$,

$$
\frac{\partial \pi}{\partial x}=\frac{\varrho}{2 m^{2}} \frac{\partial}{\partial x}\left(\hbar \frac{\partial P_{2}}{\partial x}-P_{2}^{2}\right) \frac{\varrho}{m} \frac{\partial W_{\pi}}{\partial x}
$$

and because $P_{2}$ depends on the density, $\varrho$, by the relation (see ref. ${ }^{6}$, Eq. (4.3))

$$
P_{2}=-\frac{1}{2} \hbar \cdot \partial \log \varrho / \partial x
$$

we can get the alternative expression for $\partial \pi / \partial x$,

$$
\frac{\partial \pi}{\partial x}=-\frac{\varrho}{2 m^{2}} \frac{\partial}{\partial x}\left(\frac{\hbar^{2}}{4 \varrho^{2}}\left(\frac{\partial \varrho}{\partial x}\right)^{2}-\frac{\hbar^{2}}{2 \varrho} \frac{\partial^{2} \varrho}{\partial x^{2}}\right) .
$$

We recall the expression for $W_{\mathrm{F}}$ Eq. (23) and we now see why it is possible to get the pure classical situation by modifying the potential with the addition $W_{\mathrm{F}}$. If we use the symbol $W_{\pi}$ to denote the pressure gradient potential per unit density then $W_{\pi}=-W_{\mathrm{F}}$ and the effect of adding $W_{\mathrm{F}}$ to $W$ was to subtract off the interaction between adjacent paths due to the pressure $\pi$. Equations (35) or (37) constitute the mathematical statement of the "probability pressure concept". Expressed otherwise, this concept means that quantum processes are in all essentials classical flow processes describable by classical dynamics with the additional idea that the flowing probability exerts a local pressure as would any material fluid.

If this analogy is to be shown to be complete we shall need to demonstrate the truth of certain other classical relations to which $W_{\pi}$ will need to conform. In order to show that $W_{\pi}$ really can be regarded as an addition to the external potential $W$ and really is part of a total potential $W+W_{\pi}$ which determines the behaviour of a system which is otherwise classical, we shall need to show:

A) The equation of motion can be put into the form

$$
\frac{\mathrm{d} P_{1}}{\mathrm{~d} t}=-\frac{\partial}{\partial x}\left(W+W_{\pi}\right),
$$

(38) is our first requirement. Further if we take a path defined by

$$
m \frac{\mathrm{d} x}{\mathrm{~d} t}=P_{1},
$$

and take the kinetic energy to be $T=P_{1}^{2} / 2 m$, we see immediately from (30) and (33) that

$$
E_{1}=P_{1}^{2} / 2 m+W+W_{\pi},
$$

but, if we want the classical analogue to hold completely, we shall need also to show that:

B) The path integral taken along (39) (see ref. 4 and 5 ) involves an action integral

$$
\int_{t_{\mathrm{A}}}^{t_{\mathrm{B}}} L \mathrm{~d} t=\int_{t_{\mathrm{A}}}^{t_{\mathrm{B}}}\left\{T-\left(W+W_{\pi}\right)\right\} \mathrm{d} t
$$

This is our second requirement. Let us first deal with condition (A). If we differentiate (39) along a path defined by the local velocity $\left(P_{1} / m\right)$, we get

$$
m \frac{\mathrm{d}^{2} x}{\mathrm{~d} t^{2}}=\frac{\partial P_{1}}{\partial t}+\frac{P_{1}}{m} \frac{\partial P_{1}}{\partial x}=-\frac{\partial E_{1}}{\partial x}+\frac{\partial}{\partial x}\left(\frac{P_{1}^{2}}{2 m}\right) .
$$

This step follows from ref. 1, Eq. (III.30). From (32) we now get

$$
m \frac{\mathrm{d}^{2} x}{\mathrm{~d} t^{2}}=-\frac{\partial}{\partial x}\left(\frac{P_{2}^{2}}{2 m}-\frac{\hbar}{2 m} \frac{\partial P_{2}}{\partial x}-W\right)
$$

and thus from (35)

$$
\frac{\mathrm{d} P_{1}}{\mathrm{~d} t}=-\frac{\partial}{\partial x}\left(W+W_{\pi}\right) .
$$

Thus we have indeed got condition (A).

In order to deal with condition (B), we need to return to our wave functional (16). Let us evaluate the phase $\theta(\mathrm{A} \mid \mathrm{B})$ along the real path (39). We have

$$
\begin{aligned}
\hbar \theta(\mathrm{A} \mid \mathrm{B}) & =+\operatorname{Re}\left[\int_{x\left(t_{\mathrm{A}}\right)}^{x\left(t_{\mathrm{B}}\right)} P \mathrm{~d} x-\int_{t_{\mathrm{A}}}^{t_{\mathrm{B}}} E \mathrm{~d} t\right] \\
& =\operatorname{Re}\left[\int_{t_{\mathrm{A}}}^{t_{\mathrm{B}}}\left(\frac{P P_{1}}{m}-E\right) \mathrm{d} t\right]
\end{aligned}
$$

taken along (39). If we now substitute for $E$ by (21), remembering that $P$ is a complex function $P=P_{1}$ $+i P_{2}$, we get

$$
\begin{aligned}
\hbar \theta= & \operatorname{Re}\left[\int _ { t _ { \mathrm { A } } } ^ { t _ { \mathrm { B } } } \left\{\frac{\left(P_{1}+i P_{2}\right) P_{1}}{m}\right.\right. \\
& \left.\left.-\left(\frac{\left(P_{1}+i P_{2}\right)^{2}}{2 m}-\frac{i \hbar}{2 m} \frac{\partial\left(P_{1}+i P_{2}\right)}{\partial x}+W\right)\right\} \mathrm{d} t\right] \\
= & \int_{t_{\mathrm{A}}}^{t_{\mathrm{B}}}\left[\frac{P_{1}^{2}}{2 m}+\frac{P_{2}^{2}}{2 m}-\frac{\hbar}{2 m} \frac{\partial P_{2}}{\partial x}-W\right] \mathrm{d} t \\
= & \int_{t_{\mathrm{A}}}^{t_{\mathrm{B}}}\left[T-\left(W+W_{\pi}\right)\right] \mathrm{d} t
\end{aligned}
$$

by Eq. (35). 
Thus we have also confirmed condition (B), Eq. (41).

\section{Conclusion}

This work shows very clearly some aspects of the basic structure of quantum mechanics in terms which are very closely analogous to classical hydrodynamics. In this way we are able to understand why the functional integral in quantum theory has not, so far, proved very useful. The reason for this failure is that the paths of importance for quantum theory, although in a sense deterministic and even smooth, involve a complicated self adjusting effect through the pressure potential $W_{\pi}$. Up to date no method of calculating these paths explicitly has been devised. If these paths could be calculated the functional integral could be used in something more than just the descriptive sense. However, our work shows that conventional quantum mechanics as expressed by the Schrödinger equation is essentially a subbranch of hydrodynamics, except that the quantum 'probability pressure' is a more complicated feature than is pressure in simple flow situations. It should be remarked that, although hydrodynamical processes are more directly understandable in intuitive terms than are quantum processes, hydrodynamical problems are not in general particularly easy anyway. However, the motivation for this investigation and related works has been to show up relations between apparently diverse subjects and throw some light on fundamental concepts. It is to be hoped that further study of the hydrodynamical analogy will ultimately lead to the development of computational techniques.

\section{Acknowledgement}

I am grateful to Professor M. S. Bartlett, F. R. S. and Professor I. C. Percival for some conversations which have been helpful in formulating these ideas. 